\title{
Nominalisations statives et transfert aspectuel : quel héritage sémantique ? $^{1}$
}

\author{
Richard Huyghe \\ Université Paris Diderot - Paris 7, EA 3967 CLILLAC-ARP \\ rhuyghe@eila.univ-paris-diderot.fr \\ Anne Jugnet \\ Université Paris Diderot - Paris 7, EA 3967 CLILLAC-ARP \\ anne.jugnet@univ-paris-diderot.fr
}

\section{Introduction}

Ce travail porte sur les noms dérivés statifs qui ont un corrélat morphologique dans le domaine verbal. Nous étudions les noms suffixés (en -tion et -ment notamment) qui décrivent des situations statives, et que l'on peut apparenter morphologiquement à un verbe. Sont ainsi considérées des paires comme possessionposséder, admiration-admirer, énervement-énerver, amusement-amuser, etc.

Les noms dérivés statifs sont le plus souvent construits sur des bases adjectivales, et étudiés en tant que tels. En effet, un certain nombre de suffixes nominaux opèrent régulièrement sur des adjectifs pour produire des noms de propriétés ou d'états, à l'instar des -ité, -itude, -esse, -ie, -ise, que l'on trouve dans fragilité, solitude, tendresse, jalousie, franchise (cf. Kerleroux, 2008). La fidélité aspectuelle des noms à leurs bases adjectivales, implicitement ou explicitement admise, permet généralement d'établir un parallèle sémantique entre l'adjectif et le nom qui en dérive.

Le cas des noms statifs en lien avec des prédicats verbaux apparaît comme plus marginal, quoiqu'il fasse l'objet d'une attention particulière dans plusieurs travaux récents (cf. Anscombre, 2003, Kelling, 2003, Meinschaefer, 2003, Beauseroy, 2009, Fradin, 2009, Haas, 2009). Nous souhaitons aborder l'analyse de ces noms en nous demandant si, à l'image de ce qu'on observe pour les déadjectivaux, il existe une forme de transfert aspectuel entre verbe et nom statif apparenté. L'opération de dérivation morphologique préserve-t-elle les propriétés aspectuelles de la base ? Peut-elle au contraire s'accompagner d'un changement de catégorie aspectuelle ? La sélection du suffixe joue-t-elle un rôle dans la détermination de l'aspect des nominalisations statives?

Après avoir rappelé l'existence de différents schémas de correspondance entre verbes et noms statifs, selon que les propriétés aspectuelles du nom construit semblent ou non héritées du verbe corrélé, nous nous attarderons sur les cas d'apparente discordance. Faut-il en l'occurrence considérer que le nom est dérivé du verbe ou d'un prédicat adjectival apparenté ? Comment expliquer en dernier ressort le lien sémantique, direct ou indirect, entre le verbe et le nom correspondant? Peut-on prédire quels types de verbes sont susceptibles de s'associer à des nominalisations statives? En toile de fond, nous nous interrogerons sur la permanence et la transmission du sens entre les différentes catégories grammaticales.

\section{Différents schémas de transfert aspectuel}

L'examen des paires $\mathrm{N}$ statif/ V apparenté conduit à distinguer trois cas de figure, selon que les propriétés aspectuelles des noms peuvent ou non être considérées comme (totalement ou partiellement) héritées des verbes correspondants.

\subsection{Fidélité aspectuelle}

Certains noms dérivés statifs ont pour corrélats verbaux des prédicats statifs. Tel est le cas de noms comme appartenance, connaissance, possession, persistance, (co)existence, signification, prédominance. 
La stativité de ces noms explique qu'ils ne soient compatibles ni avec des verbes supports dynamiques (e.g. faire, effectuer, procéder à), ni avec des prédicats événementiels (e.g. avoir lieu, se produire, se dérouler, cf. Haas et al., 2008) :

(1) a. *Ils ont (fait / effectué / procédé à) (une possession / une connaissance / une appartenance / une signification)

b. *(Une possession / une connaissance / une appartenance / une signification) (a eu lieu / s'est produite / s'est déroulée) à Paris la semaine dernière

Quant aux verbes correspondants, leur incompatibilité avec la forme progressive être en train de témoigne de leur aspect non dynamique :

(2) *Pierre est en train de (posséder une maison / connaître l'anglais / appartenir à une confrérie de moines trappistes)

On peut ainsi établir un parallèle entre la situation dénotée par le verbe et ses arguments et celle exprimée par le nom correspondant et ses compléments :
a. Pierre appartient à cette confrérie / l'appartenance de Pierre à cette confrérie
b. Elle connaît parfaitement l'anglais / sa connaissance parfaite de l'anglais
c. Il existe cinq types de nuages / l'existence de cinq types de nuages
d. Le problème persiste / la persistance du problème

Les noms considérés ici sont donc fidèles, du point de vue aspectuel, aux verbes auxquels ils sont apparentés. Ce constat appelle trois remarques.

La première est qu'on peut se demander si, parmi ces noms, ceux qui se finissent par -ance ne sont pas, dans certains cas au moins, construits sur des bases adjectivales en -ant et suffixés par-ce. Ce schéma de construction pourrait s'appliquer à persistance et prédominance, étant donné l'attestation des adjectifs persistant et prédominant. Mais il est plus difficile de l'envisager pour des noms comme connaissance et appartenance, qui seront plutôt considérés comme d'authentiques déverbaux. Que l'on opte ou non pour un traitement unitaire des noms statifs en -ance dotés d'un corrélat verbal, la question de la préservation de l'aspect est tranchée : le nom, déverbal ou déadjectival, est fidèle à la fois à sa base morphologique et au verbe corrélé, en ce qu'il exprime une propriété individuelle du sujet — nom, verbe et éventuel adjectif constituent tous des « individual level predicates" (Carlson, 1980). Autrement dit, dans tous les cas, l'aspect des noms en -ance peut être considéré comme hérité des verbes apparentés, et l'opération de dérivation est de ce point de vue transparente.

Une autre remarque concerne la polysémie des noms statifs analysés, dont beaucoup sont susceptibles d'avoir une acception non marquée aspectuellement, comme dans :
a. Pierre aime faire étalage de ses connaissances
b. Quelle est la signification de ce mot?
c. En 211 avant J.-C., Carthage doit se séparer de ses possessions espagnoles

Les noms considérés ici ont, par métonymie, la capacité de dénoter un objet ou un contenu caractérisé par la propriété décrite dans l'acception stative ${ }^{2}$. Leurs traits aspectuels sont alors neutralisés. Cette double interprétation des noms statifs est comparable à celle des noms dynamiques susceptibles de dénoter des actions ou des résultats (e.g. la construction du barrage par EDF vs une construction solide, cf. Grimshaw, 1990, Pustejovsky, 1995, Alexiadou, 2001).

Notons enfin que l'on trouve, parmi les cas de fidélité aspectuelle, des exemples de double acception stative-dynamique, le nom étant alors lié à un verbe polysémique. Tel est le cas de adorer-adoration, concorder-concordance, correspondre-correspondance :

a. Lisa (adore / *est en train d'adorer) son frère / l'adoration de Lisa pour son frère

b. A son retour, Moïse trouve les Israélites en train d'adorer un veau / l'adoration d'un veau par les Israélites 
Comme précédemment, l'aspect des noms sous (5) peut être envisagé comme directement hérité des verbes auxquels ils s'apparentent, en vertu de leur correspondance sémantique immédiate.

\subsection{Les nominalisations partielles}

Certains noms dérivés statifs sont liés à des verbes qui ont une double lecture d'état ou d'activité, mais, contrairement aux noms comme adoration, ils se cantonnent à l'interprétation stative. Tel est le cas d'admiration, considération, ignorance et vénération.

Les verbes admirer, considérer, ignorer, vénérer peuvent en effet exprimer des actions, puisqu'ils peuvent se construire avec être en train de, être repris dans une pseudo-clivée par le verbe faire, ou figurer dans l'expression voir $x V(y)$ (cf. Gross, 1975, Ruwet, 1995, Martin, 2008 inter alia) :

(6) a. Sophie (admire / est en train d'admirer) le paysage

b. Ce que fait Sophie, c'est admirer le paysage

c. J'ai vu Sophie admirer le paysage

Mais ils ont également une lecture stative, dans laquelle ils constituent des prédicats de propriété, incompatibles avec les trois tests mis en œuvre dans (6) :
a. Vincent (admire / ??est en train d'admirer) l'honnêteté
b. ??Ce que fait Vincent, c'est admirer l'honnêteté
c. ??J'ai vu Vincent admirer l'honnêteté

Cette polysémie verbale ne s'observe pas dans le domaine nominal. Les noms correspondants n'admettent que l'interprétation stative. En effet, ils n'ont pas de structure argumentale dynamique (i.e. comportant un argument agent introduit par par, cf. Kelling, 2003), ne se construisent pas avec les verbes supports dynamiques, ne peuvent pas être modifiés par en cours (cf. Anscombre, 2005), ni être le sujet de avoir lieu, se produire ou se dérouler :
a. *l'admiration du paysage par Sophie
b. *Marc a (fait / effectué / procédé à) une admiration hier
c. *Il y a une admiration en cours au sommet de la montagne
d. *Son admiration (a eu lieu / s'est produite / s'est déroulée) la semaine dernière

En revanche, les noms comme admiration peuvent se combiner avec un verbe support statif, compléter le nom sentiment, et intégrer dans leur structure argumentale un argument patient introduit par pour (cf. Anscombre, 1995, Flaux \& Van de Velde, 2000) :
a. Vincent (éprouve / ressent) de l'admiration pour son frère
b. un sentiment d'admiration
c. l'admiration de Vincent pour son frère

Ne sélectionnant qu'une acception du verbe apparenté, les nominalisations du type admiration peuvent être qualifiées de "partielles » (Haas et al., 2008). On peut se demander sur quelles bases se fait cette sélection aspectuelle. Qu'elle soit due au suffixe paraît peu vraisemblable, puisque pour certains noms au moins (ceux en -tion), le suffixe ne comporte pas de spécification aspectuelle stative. D'une part, le suffixe -tion peut générer des noms dynamiques (e.g. réparation, destruction, imitation). D'autre part, des noms suffixés en -tion sont tout à fait capables de reproduire la double acception du verbe corrélé, comme on l'a vu dans le cas d'adoration. ${ }^{3}$

L'hypothèse que nous faisons concernant les nominalisations partielles est que celles-ci obéissent à une prédisposition sémantique du verbe : elles sélectionnent un statut aspectuel du verbe établi «par défaut ». Selon cette hypothèse, une nominalisation partielle pourrait être considérée comme un révélateur de la signification principale du verbe qui lui correspond. 


\subsection{Des cas de décalage aspectuel}

Les noms examinés jusqu'ici sont fidèles, ne serait-ce que partiellement, aux verbes auxquels ils s'apparentent, i.e. leur aspect statif est déjà présent dans le domaine verbal. Ce constat ne vaut toutefois pas pour tous les noms construits non dynamiques. En effet, des décalages aspectuels entre verbes et noms apparentés s'observent dans toute une série de cas, tels que s'acharner-acharnement, affoleraffolement, agacer-agacement, amuser-amusement, attendrir-attendrissement, découragerdécouragement, émerveiller-émerveillement, s'entêter-entêtement, énerver-énervement, exaspérerexaspération, irriter-irritation, motiver-motivation, se recueillir-recueillement, se résigner-résignation. En l'occurrence, les noms sont statifs, puisqu'ils peuvent compléter les noms sentiment ou état, les verbes éprouver, ressentir ou faire preuve de (Kerleroux, 2008) :

(10) a. un sentiment (d'agacement / de découragement / de recueillement)

b. un état (d'affolement / de motivation / de résignation)

c. (éprouver / ressentir) (de l'exaspération / de l'irritation / de l'amusement)

d. Il a fait preuve (d'acharnement / d'énervement / d'entêtement)

Mais les verbes correspondants sont dynamiques, comme en témoigne leur compatibilité avec être en train de, leur reprise par faire et leur construction dans la tournure voir $x V(y)$ (cf. Meinschaefer, 2003, Martin, 2008) :

(11) a. Il est en train de (s'entêter / irriter ses employés / d'exaspérer tes parents / s'acharner)

b. Ce que fait Sophie, c'est (amuser les enfants / agacer sa sœur / se recueillir devant le monument / énerver son entourage)

c. J'ai vu Vincent (affoler les défenseurs du camp adverse / motiver ses troupes / décourager ses collègues / se résigner)

Il faut toutefois distinguer deux cas ici. D'un côté, certains verbes, dont les pronominaux (s'acharner, s'entêter, se recueillir), ont un sujet qui est toujours agentif, et ils sont donc clairement dynamiques. De l'autre, des verbes comme amuser, décourager, exaspérer, s'ils sont compatibles avec des sujets agentifs, peuvent également avoir une lecture non agentive, comme dans :

a. Cette situation (amuse / énerve / agace) Marion

b. Tous ces projets (motivent / exaspèrent / découragent) nos partenaires

Les exemples sous (12) sont incompatibles avec des adverbes d'intentionnalité orientés vers le sujet (e.g. délibérément, volontairement, intentionnellement, cf. Dowty, 1979, Verkuyl, 1989). La possibilité d'avoir un sujet non agentif explique l'ambiguité de phrases comme :

Pierre (m'amuse / m'agace / me décourage)

Le sujet ici peut être vu comme un agent ou comme une simple cause, dénuée de toute intentionnalité.

On peut se demander si dans les emplois du type (12), les verbes sont encore dynamiques. Rappelons d'abord que si l'agentivité implique la dynamicité, l'inverse n'est pas vérifié : il existe des situations dynamiques dans lesquelles le sujet n'est pas un agent (e.g. Les feuilles tombent de l'arbre). Il est vrai que les verbes comme amuser, décourager, énerver dans leur emploi non agentif se combinent moins facilement avec être en train de :

(14) a. (?)Cette situation est en train d'(amuser / énerver / agacer) Marion

b. (?)Tous ces projets sont en train de (motiver / décourager / exaspérer) nos partenaires

De même, les tests de la reprise par faire et de la construction en voir $x V(y)$ donnent des résultats mitigés :

(15) a. ?Ce que fait cette situation, c'est (amuser / énerver / agacer) Marion

b. ?Ce que font tous ces projets, c'est (motiver / décourager / exaspérer) nos partenaires

(16) a. (?)J'ai vu cette situation (amuser / énerver / agacer) Marion

b. (?)J'ai vu tous ces projets (motiver / décourager / exaspérer) nos partenaires 
Ces données doivent être nuancées, notamment dans (15). Faire en effet s'emploie préférentiellement avec des sujets agentifs, et cette tendance peut parasiter le test mobiliséf. La tournure voir $x V(y)$ semble mieux s'appliquer, quoiqu'elle paraisse moins naturelle que dans (11c).

Néanmoins, il est difficile de conclure à la stativité des emplois non agentifs d'amuser, décourager, énerver, etc. Il y a de fait une différence importante entre ces verbes et ceux précédemment analysés. D'une part, posséder, appartenir, connaître, admirer (dans son acception stative), etc. récusent nettement les trois tests de dynamicité employés ici. D'autre part, les verbes du type amuser peuvent tout à fait, dans leur lecture non agentive, s'employer comme prédicats de phrases événementielles :
a. Le spectacle a beaucoup amusé les enfants
b. L'ampleur de la tâche nous a découragés
c. La défaite de leur équipe les a vraiment énervés
d. Le jeu de l'acteur principal a exaspéré tout le public

En effet, les phrases sous (17) peuvent répondre à la question Que s'est-il passé ? et donc constituer des descriptions événementielles. Ce type d'interprétation, qui implique un ancrage spatio-temporel et est caractéristique des verbes dynamiques, échappe aux verbes posséder, appartenir, connaître, etc.

Il y a bien selon nous une dynamicité des verbes amuser, décourager, énerver, etc., même si à certains égards celle-ci n'est pas prototypique. En tout état de cause, la double lecture agentive / non agentive des verbes comme amuser n'est pas de nature à fonder une polysémie semblable à celle d'admirer, ignorer ou considérer. Les noms correspondant aux verbes du type amuser ne peuvent pas être considérés comme des nominalisations partielles, i.e. comme calqués aspectuellement sur une partie seulement des acceptions du verbe corrélé. De fait, contrairement à admiration, les noms amusement, découragement, énervement peuvent correspondre au résultat d'une situation dynamique :

(18) a. Sophie est en train d'admirer le paysage. *Son admiration est visible

b. Pierre est en train de (amuser/ décourager/ énerver) ses collègues. Leur (amusement / découragement / énervement) est perceptible ${ }^{5}$

Nous reviendrons sur les particularités aspectuelles des verbes considérés ici, mais nous pouvons dire d'emblée que les paires qu'ils forment avec leurs noms apparentés ne sont pas réductibles à des cas d'héritage aspectuel complet ou partiel. Nous considérerons donc qu'il y a ici un décalage aspectuel entre un verbe (essentiellement) dynamique et son corrélat nominal statif. On peut se demander comment rendre compte, à la fois sémantiquement et morphologiquement, d'un tel décalage.

\section{Des noms déverbaux ou déadjectivaux?}

Dans les cas d'apparente discordance aspectuelle, la divergence sémantique entre le nom et le verbe conduit à s'interroger sur la teneur de l'opération de dérivation. Le phénomène observé en 2.3 pourrait s'expliquer par le fait que les noms ne sont pas directement dérivés des verbes correspondants, mais plutôt d'adjectifs issus de leurs participes passés. Des noms comme irritation, motivation, découragement, énervement seraient ainsi dérivés des participes passés adjectivaux irrité, motivé, découragé, énervé, et non des verbes irriter, motiver, décourager, énerver. Les noms en question paraissent en effet plus proches sémantiquement de formes adjectivales que verbales. Déadjectivaux, ils préserveraient l'aspect de leur base, et serait ainsi résolu le problème de la différence d'aspect entre noms et verbes apparentés.

\subsection{L'hypothèse d'une dérivation adjectivale}

L'idée que les noms psychologiques comme irritation, découragement, etc. sont dérivés de prédicats adjectivaux est défendue par Van de Velde (1995) et Van de Velde \& Flaux (2000). Cette hypothèse se fonde principalement sur des arguments syntactico-sémantiques. De fait, le nom est, comme l'adjectif, doté d'une structure argumentale à une place renvoyant à l'expérienceur de l'état décrit. Cette structure 
argumentale ne peut en aucun cas intégrer un agent, ce en quoi le nom se distingue du verbe auquel il s'apparente :
a. Marion a irrité le directeur
b. *l'irritation du directeur par Marion
c. \#l'irritation de Marion

a. Le directeur est irrité / un directeur irrité

b. l'irritation du directeur

(21) a. Le jury a découragé les candidats

b. *le découragement des candidats par le jury

c. \#le découragement du jury

(22) a. Les candidats sont découragés / des candidats découragés

b. le découragement des candidats

Ce parallélisme entre adjectif et nom permet-il de conclure à une dérivation morphologique ? L'hypothèse déadjectivale est guidée par un postulat implicite, celui de la préservation de l'aspect dans les opérations de dérivation: la structure thématique et argumentale du nom étant similaire à celle de l'adjectif, le premier dérive du second. Corollairement, le suffixe est considéré comme transparent, i.e. neutre du point de vue aspectuel. On peut s'interroger sur les implications d'une telle conception.

\subsection{Le rôle du suffixe}

Pour la théorie, un grand avantage de l'hypothèse déadjectivale est la transparence sémantique de la dérivation : il n'y a pas à charger l'opération de dérivation d'une quelconque conversion aspectuelle. Le suffixe, en particulier, peut être considéré comme neutre en ce qu'il calque l'aspect du nom dérivé sur celui de sa base. Se pose toutefois la question de la catégorie grammaticale de la base sélectionnée.

En effet, les suffixes -ment et -tion présents dans les noms recensés en 2.3 ont ceci d'intéressant qu'ils peuvent être associés à des noms statifs (exaspération, signification, dévouement, affolement) et dynamiques (réparation, abolition, licenciement, affrontement). Dans les cas où les noms sont fidèles aspectuellement aux verbes qui leur sont apparentés (e.g. réparation, signification, licenciement), il n'y a aucun inconvénient à les considérer comme dérivés de ces verbes. L'hypothèse déadjectivale s'intègre tout à fait dans ce schéma de dérivation fidèle. Mais on se retrouve alors, pour chaque suffixe, avec deux règles de construction distinctes : le -(t)ion de signification, possession, réparation, abolition opérerait sur des bases verbales statives (signifier, posséder) ou dynamiques (réparer, abolir), tandis que le -tion de motivation, irritation, exaspération, exclusivement statif, se construirait sur des participes passés adjectivaux. De son côté, le -ment de licenciement, harcèlement, lancement opérerait sur des verbes dynamiques, tandis que le -ment de dévouement, découragement, émerveillement sélectionnerait des bases adjectivales statives. Ces deux doubles règles sont représentées ci-dessous :

$$
\begin{aligned}
& - \text { tion } \\
& \mathrm{V}+\text {-tion }>\mathrm{N}(\text { aspect } \mathrm{N}=\text { aspect } \mathrm{V}=[ \pm \mathrm{dyn}]) \\
& \text { Adj }+ \text {-tion }>\mathrm{N}(\text { aspect } \mathrm{N}=\text { aspect } \mathrm{Adj}=[-\mathrm{dyn}]) \\
& - \text { ment } \\
& \mathrm{V}+- \text { ment }>\mathrm{N}(\text { aspect } \mathrm{N}=\text { aspect } \mathrm{V}=[+\mathrm{dyn}]) \\
& \text { Adj }+ \text {-ment }>\mathrm{N}(\text { aspect } \mathrm{N}=\text { aspect } \mathrm{Adj}=[-\mathrm{dyn}])
\end{aligned}
$$

L'hypothèse de la dérivation adjectivale implique donc l'existence de deux nouvelles règles de construction, certes transparentes du point de vue aspectuel, mais imposant une sélection sur la catégorie de la base différente de celle traditionnellement envisagée. On peut s'interroger sur la pertinence de ces règles, ainsi que sur leur coût théorique, notamment si l'existence de suffixes -tion et -ment « statifs », i.e. opérant sur des bases adjectivales, n'est pas repérée ailleurs dans le lexique que dans le cas des adjectifs verbaux. 
Kerleroux (2008) se penche sur la productivité du suffixe -tion et sur son application aux adjectifs, dont certains n'ont pas de forme verbale corrélée (e.g. adéquat-adéquation, abject-abjection, discretdiscrétion, précis-précision, concis-concision, dévot-dévotion, cf. aussi Dell, 1979). Sans trancher la question de savoir si le suffixe déadjectival est ou non synchroniquement productif en français, Kerleroux émet l'idée que les noms de propriétés en -tion pourraient relever d'un phénomène de morphologie non constructionnelle, consistant en un emprunt massif au latin, lui-même susceptible d'être réanalysé comme une dérivation en français ${ }^{6}$. Le fait est que, du point de vue statistique, la dérivation verbale en -tion est productive et représentative, quand la dérivation purement adjectivale, si elle existe, reste très marginale. Le constat est plus net encore dans le cas du suffixe -ment, puisque les noms en -ment construits sur des adjectifs sans corrélat verbal sont très rares (e.g. dénué-dénuement, désoeuvré-désoeuvrement, enneigéenneigement). L'argument statistique peut être opposé à l'hypothèse déadjectivale : faut-il envisager l'existence d'un suffixe spécifique, i.e. doté d'une règle de construction propre, si celui-ci est essentiellement destiné à rendre compte des cas où noms et verbes corrélés divergent aspectuellement ?

Une autre difficulté pour l'hypothèse déadjectivale est d'expliquer la relation sémantique existant entre les différentes acceptions d'un même nom en -tion ou -ment. Certaines nominalisations peuvent en effet avoir une double lecture, stative ou dynamique, alors que le verbe apparenté est univoquement dynamique (cf. Gross \& Kiefer, 1995). Tel est le cas de démobilisation, humiliation, épuisement, chamboulement, etc., qui dénotent des états dans (25) et des actions dans (26) :

(25) a. ressentir (une grande humiliation / un grand chamboulement)

b. un état (d'épuisement / de démobilisation)

(26) a. l'humiliation de Vincent par son frère

b. l'épuisement des ressources pétrolières par les pays occidentaux

c. Il faut procéder à la démobilisation des troupes

d. Un véritable chamboulement a eu lieu dans l'industrie du disque

Dans l'hypothèse déadjectivale, les deux emplois doivent être appréhendés comme des cas d'homonymie, puisqu'ils résultent de schémas de construction différents - impliquant la sélection de bases de catégories différentes et la construction de noms d'aspects différents (cf. Kerleroux, 2008, Beauseroy, 2009). Une telle option, qui risquerait d'occulter le lien de sens existant entre les lectures dynamique et stative des noms concernés, est sémantiquement insatisfaisante. En outre, une conception homonymique des noms sous (25)-(26) les mettrait sur le même plan qu'un nom comme correction, tel qu'il est employé dans (27):

a. La correction des copies nous a pris plus longtemps que prévu

b. Pierre est d'une correction exemplaire

Correction dans (27a) est clairement dérivé du verbe corriger, dont il préserve l'aspect dynamique, tandis que dans (27b), il est sémantiquement lié à l'adjectif correct (cf. Kerleroux, 2008). Or il y a ici une nette différence entre les deux formes nominales, qui n'apparaît pas dans (25)-(26). En effet, des noms comme humiliation, dans leurs emplois statifs et dynamiques, peuvent attribuer le même rôle sémantique - celui d'expérienceur - à leur complément en de (e.g. l'humiliation des détenus par leurs geôliers vs L'humiliation des détenus était visible). Un nom comme correction, par contre, selon qu'il dénote une action ou une propriété, se voit associer des programmes de complémentation bien distincts. Ainsi le complément en de du correction dynamique ne peut pas dénoter un humain, contrairement à ce qui est le cas pour le correction statif. Un argumentaire possible, pour les tenants de l'hypothèse déadjectivale, serait d'insister sur l'origine verbale de la base adjectivale sélectionnée dans (26). Cela reviendrait toutefois à reconnaître que le lien avec la catégorie verbale, fût-il indirect, est déterminant dans la dérivation de noms statifs en -tion et -ment.

Une dernière question se pose, en creux, concernant les noms du type irritation et découragement: s'il s'agit de noms statifs déadjectivaux, pourquoi le suffixe qu'ils sélectionnent n'est-il pas l'un de ceux spécifiquement dédiés aux états et exclusivement construits sur des adjectifs, tels que -ité, -esse, -ance, itude, etc. ? Là encore, on peut faire valoir que -tion et -ment ont pour particularité de sélectionner des 
adjectifs eux-mêmes issus de participes passés, mais cela implique que le caractère indirectement verbal de la base est pris en compte dans l'opération de dérivation. D'où l'idée récurrente que les suffixes -tion et -ment, en tant qu'affixes productifs en français, présentent une affinité particulière avec la catégorie verbale.

\subsection{L'hypothèse d'une dérivation verbale}

$\mathrm{Au}$ vu des difficultés posées par l'hypothèse déadjectivale, et pour des raisons qui seront précisées ensuite, tenant à l'existence d'une certaine continuité sémantique entre le verbe dynamique et le nom statif correspondant, nous privilégierons l'idée que les noms exaspération, motivation, découragement, énervement, etc. sont des déverbaux. Un des avantages de cette hypothèse est, à nos yeux, d'inciter à s'interroger sur la nature du transfert aspectuel lorsque celui-ci s'accompagne d'un changement de catégorie grammaticale. Il nous semble en effet qu'une comparaison aspectuelle entre verbes et noms s'impose s'il existe entre eux une parenté de forme - que la dérivation soit directe ou indirecte. Quand bien même on envisagerait le passage par une étape adjectivale, le décalage observé entre verbes dynamiques et noms corrélés statifs n'est pas arbitraire : il concerne un certain type de paires verbonominales et doit en tant que tel faire l'objet d'une analyse.

Nous réduirons donc, pour motiver notre enquête, -tion, -ment à un schéma de dérivation unique :

$$
\begin{aligned}
& \text {-tion /-ment } \\
& \mathrm{V}+\text {-tion / -ment }>\mathrm{N}
\end{aligned}
$$

Selon cette hypothèse, les suffixes -tion et -ment se construisent toujours sur des verbes, qu'ils produisent des noms statifs ou dynamiques, fidèles ou non aspectuellement à leurs bases. Ces deux suffixes sont fondamentalement liés à la catégorie verbale de la base sélectionnée.

\section{La sélection aspectuelle, du verbe au nom}

Si les noms recensés en 2.3 sont effectivement des déverbaux, issus de verbes dynamiques, comment expliquer leur caractère statif? Comment s'opère le transfert aspectuel du verbe au nom qui en dérive, et quelles sont les contraintes sur la dérivation de noms statifs à partir de bases verbales dynamiques? Notre hypothèse est que la nominalisation peut sélectionner un trait statif présent dans l'entrée lexicale du verbe (laissant à l'arrière-plan sa composante dynamique), et indique qu'un état est saillant dans le sémantisme du verbe.

Deux cas seront examinés successivement, selon que le nom provient (i) d'un verbe qui décrit une action visant à mettre dans un certain état un objet expérienceur (e.g. décourager, énerver, émerveiller), ou (ii) d'un verbe qui décrit une action dont le stimulus est un état du sujet agissant (e.g. s'acharner, s'entêter, se dévouer).

\subsection{Les noms issus de verbes causatifs d'état}

Les noms examinés ici dérivent de verbes qui expriment une action dont le but est de conférer un certain état psychologique au référent de l'objet (ou de l'objet-sujet, dans le cas des réfléchis tels que s'affoler, s'amuser ou s'énerver) (cf. Ruwet, 1972, Anscombre, 1995, Van de Velde, 1995, Meinschaefer, 2003 inter alia). Les verbes en question incluent donc dans leur structure aspectuelle une composante stative ; c'est sur cette composante que focalisent leurs nominalisations.

\subsubsection{Propriétés des bases verbales}

Tous les types de verbes dynamiques ne sont pas associés à des nominalisations statives : au premier chef sont concernés les causatifs d'état. La composante stative de ces verbes, i.e. l'état résultant de l'action décrite, est saillante en ce sens qu'elle constitue l'objectif de l'action. Elle peut dans certains cas 
déterminer le telos, i.e. le terme intrinsèque de l'action, ce qui explique la compatibilité des verbes avec les compléments de temps introduits par en :

$$
\text { Sophie (a apaisé / a attendri / a découragé / a motivé / a affolé) son frère en dix minutes }
$$

Ces mêmes verbes connaissent avec les compléments en pendant une lecture ambiguë, selon que la durée couvre l'action du sujet ou simplement l'état qui en résulte (cf. Binnick, 1969, Dowty, 1979) :

\section{(30) Sophie (a apaisé / a attendri / a découragé / a motivé / a affolé) son frère pendant dix minutes}

Cette double interprétation est exclue pour des verbes comme construire, lire ou manger. Elle s'appuie sur la saillance de la composante stative dans la structure événementielle des verbes apaiser, attendrir, décourager, etc.

Autre indice de cette composante aspectuelle stative, les verbes du type amuser, énerver, décourager ne spécifient pas l'action accomplie, contrairement à l'état visé. Comme le montrent les phrases suivantes, le procès est sous-spécifié : hors contexte, il est difficile de déterminer quelle action précise a été accomplie par le sujet. Ceci explique que les phrases en (31a) et (31b) ne soient pas contradictoires, ni (31c) redondant :

(31) a. Je sais que Marie a énervé Jean, mais je ne sais pas ce qu'elle a fait précisément (vs ?Je sais que Marie a réparé le toit, mais je ne sais pas ce qu'elle a fait)

b. Tu dis que Marie a énervé Jean. Mais qu'a-t-elle fait pour l'énerver?

( vs ?Tu dis que Marie a lu ce livre. Mais qu'a-t-elle fait pour le lire ?)

c. Marie a énervé Jean (en le bousculant / en l'injuriant)

(vs ?Marie a écrit cette lettre en tenant son stylo)

L'état impliqué par ces verbes explique également leur emploi avec des adverbes de degré, comme dans :
a. Cela m'a beaucoup amusé
b. Le tenant du titre a fortement découragé ses adversaires
c. Claire a énormément agacé sa mère

Les procès décrits peuvent ainsi être caractérisés selon leur intensité, ce qui témoigne de leur affinité avec la catégorie stative. On peut mettre en relation cette «intensivité » avec le caractère massif, i.e. gradable, des nominalisations (beaucoup (d'amusement / de découragement / d'agacement)). Il y a là une marque de continuité sémantique entre le verbe et le nom. Le sémantisme des noms comme amusement et découragement doit toutefois être examiné plus précisément : conservent-ils un lien avec la dynamicité du verbe dont ils sont dérivés?

\subsubsection{Propriétés des noms dérivés}

Les noms dérivés des verbes psychologiques, quoique statifs, gardent une trace de la dynamicité du verbe. En effet, l'état décrit étant conçu comme le résultat d'un procès, il est nécessairement vu comme extrinsèque et épisodique, i.e. il ne constitue pas une propriété définitionnelle de l'expérienceur. D'où la compatibilité des noms psychologiques avec des expressions comme un moment de, une phase de, une période de, dix minutes de, deux heures de, etc. :

(33) un moment d'exaspération, une phase d'énervement, une période d'apaisement, dix minutes d'affolement, deux heures d'émerveillement

L'association de l'état à certaines situations ancrées temporellement permet au nom de préciser une modalité de l'action dans le cadre de phrases événementielles, notamment dans la tournure avec $N$ :

(34) a. Le ministre a répondu aux journalistes avec agacement

b. Les enfants regardent le clown avec amusement

c. Pierre constate avec exaspération que personne ne l'écoute 
Ici, l'état observé accompagne une action, en vertu du fait que cet état est considéré comme contingent et lui-même causé par quelque chose. Les phrases (34) sous-tendent les suivantes : Les journalistes agacent le ministre, Le clown amuse les enfants, Le fait que personne ne l'écoute exaspère Pierre.

De la même façon, dans la tournure dans son $N$, les noms psychologiques ont la capacité de dénoter à la fois une cause et un ancrage temporel (35a), ce en quoi ils se distinguent des noms de propriétés, qui admettent la lecture causale mais excluent toute interprétation temporelle (35b) (cf. Haas, 2009) :

a. Dans (son énervement / son affolement / son exaspération), il a quitté son domicile en oubliant ses clés

b. Dans (son ignorance / son avarice / son égoïsme), il méprise ceux qui l'entourent

On peut y voir de nouveau un lien avec la dynamicité exprimée par le verbe source, la dynamicité impliquant le rapport au temps. Les verbes comme ( $\left.s^{\prime}\right)$ énerver, en tant que prédicats dynamiques, s'associent à des états transitoires et les noms dérivés, en tant qu'ils décrivent ces états (i.e. des états suscités dynamiquement), véhiculent une description temporelle.

Par ailleurs, comme nous l'avons vu plus haut, certains noms psychologiques, tels que affaiblissement, apaisement, aveuglement, chamboulement, humiliation, démobilisation, envoûtement, épuisement, sont susceptibles d'avoir, en plus d'une lecture stative, une interprétation dynamique, directement héritée des verbes correspondants (cf. (25)-(26)). Ces noms se distinguent des «purs» statifs, comme affolement, agacement, attendrissement, découragement, émerveillement, enchantement, étonnement, exaspération. Aux couples (25)-(26), on peut opposer les suivants :

$$
\text { a. éprouver (de l'agacement / de l'exaspération) }
$$
a. *(l'agacement / l'exaspération) de Pierre par sa sœur
b. *Il faut procéder à (l'étonnement / l'émerveillement) de votre auditoire

Or il est possible de relier la mono- / bisémie des noms en question à certaines particularités des verbes dont ils sont dérivés. Il semble en effet que la composante dynamique soit plus saillante dans l'entrée lexicale des verbes causatifs d'état associés à des nominalisations polysémiques. Ces verbes se combinent plus facilement avec certains tests d'agentivité et de dynamicité. Ils se distinguent en particulier par leur compatibilité avec certains adverbes orientés vers l'agent, tels que patiemment, attentivement, avec zèle, et avec les structures causatives :

(38) a. Pierre a patiemment (affaibli / épuisé / humilié) ses adversaires

b. Il a fait (envoûter Marie par un sorcier/ apaiser les tensions par plusieurs diplomates / démobiliser les troupes par le général)
a. ??Pierre a patiemment (agacé / attendri / exaspéré) sa femme
b. ??Il a fait (affoler sa sœur par ses parents / émerveiller les enfants par un père Noël / étonner Marion par ses amies) ${ }^{7}$

Les noms dérivés de verbes du premier ensemble considéré, s'ils peuvent dénoter des états (résultants), conservent également la saillance du trait dynamique de leur base verbale, d'où leur double interprétation. Les particularités des noms psychologiques peuvent ainsi s'expliquer par les spécificités sémantiques des verbes apparentés; il y a bien une certaine continuité entre verbes psychologiques et noms dérivés. Corollairement, les différences entre nominalisations peuvent nous renseigner sur la structure aspectuelle des verbes dont elles sont dérivées.

\subsection{Les noms issus de verbes impliquant un état stimulus}

Les nominalisations statives examinées ici sont dérivées de verbes dénotant des actions qui, sans comprendre d'état résultant, impliquent un état ressenti par un expérienceur - l'état en question pouvant être assimilé à un stimulus. Ce cas de figure concerne des paires telles que : s'acharner-acharnement, se 
dévouer-dévouement, s'entêter-entêtement, s'empresser-empressement, s'égarer-égarement, se morfondre-morfondement, se recueillir-recueillement, se résigner-résignation.

\subsubsection{Propriétés des bases verbales}

Comme dans le cas des causatifs d'état, il semble que le sémantisme des verbes du type s'acharner laisse l'action sous-spécifiée. C'est l'état d'un sujet - état motivant l'action, ou la manière dont l'action est accomplie - qui fait l'objet d'une description spécifiée, ainsi que l'illustrent les exemples suivants :

(40) a. Je sais que Marie s'est dévouée pour sa grand-tante, mais je ne sais pas ce qu'elle a fait précisément

b. Tu dis que Marie s'est entêtée. Mais qu'a-t-elle fait précisément ?

c. Jean s'est acharné sur cette inconnue en la lardant de dizaines de coups de couteau

Certains des verbes concernés sont également compatibles avec des adverbes de degré :

(41) a. Marc s'est beaucoup dévoué pour cette cause

b. Il s'est particulièrement acharné sur ce problème

c. Je me suis fortement égaré dans mes réponses

Comme précédemment, le marquage de l'intensité dans le domaine verbal est à mettre en relation avec le caractère massif des noms dérivés (e.g. beaucoup de (dévouement / entêtement / égarement)).

Il semble en outre que les nominalisations étudiées ici soient systématiquement et univoquement statives. On peut envisager que la nominalisation stative ne soit pas concurrencée par une nominalisation dynamique en raison du statut particulier du sujet de ces verbes : celui-ci semble être conçu comme un expérienceur avant d'être un agent à l'origine d'une action. Le statut pronominal des verbes en question corrobore cette particularité.

\subsubsection{Propriétés des noms dérivés}

Comme les nominalisations des verbes causatifs d'état, les noms dérivés de s'entêter, s'acharner, s'empresser, se dévouer, etc. gardent une trace de la dynamicité de leur base. A l'instar des découragement, agacement et consorts, ils dénotent des états épisodiques. Correspondant à des situations ancrées dans le temps, ils sont compatibles avec les tournures un moment de $N$, une phase de $N$, une période de $N$, dix minutes de $N$, deux heures de $N(42)$, et peuvent spécifier une manière d'agir, dans des constituants de la forme avec $N(43)$ :

(42) un moment de morfondement, une phase d'égarement, une période de résignation, dix minutes de recueillement, deux heures d'acharnement

(43) a. Vincent a malheureusement agi avec empressement

b. Il mène tous ces combats avec acharnement

c. Sophie a accepté la situation avec résignation

Le nom, comme le verbe, ne présupposant pas une action spécifiée, il est possible de l'employer pour préciser la modalité d'une action décrite par ailleurs. Les phrases (43) sont ainsi proches sémantiquement de : Vincent s'est malheureusement empressé d'agir, Il s'acharne à mener tous ces combats, Sophie s'est résignée à accepter la situation.

Le caractère épisodique de la plupart des noms statifs analysés ici apparaît également dans l'interprétation causo-temporelle de la tournure dans son $N$ :

(44) Dans (son entêtement/ son empressement/ son égarement), Don Quichotte a confondu les troupeaux de moutons avec des armées en marche

Enfin, le lien avec la dynamicité des prédicats verbaux peut être mis en évidence dans l'expression faire preuve de $N$ (cf. § 2.3): 
Faire preuve de se construit en effet avec des noms statifs, mais qui impliquent une forme de dynamicité en ce sens qu'ils sont associés à des types de comportement, généralement instanciés dans des occurrences événementielles (e.g. faire preuve (de courage/ de sang-froid/d'inventivité)). Les noms dans (45) permettent de cibler des tendances comportementales : ils décrivent des états qui se révèlent à travers des actions, et qui se trouvent donc en lien étroit avec ce qui est exprimé par les verbes s'acharner, se dévouer et s'empresser.

\section{Conclusion}

L'examen des différentes nominalisations statives dérivées de verbes dynamiques nous mène à conclure que les discordances aspectuelles ne sont pas si conséquentes. Tout au moins peuvent-elles s'expliquer d'après la structure sémantique des verbes sources. La dérivation de noms statifs n'est en effet possible que lorsque le sémantisme de la base verbale comprend une composante stative (généralement saillante). Les nominalisations sélectionnent ainsi un trait présent dans l'entrée lexicale des verbes dont elles sont dérivées. En outre, le décalage observé entre verbes dynamiques et noms statifs doit être nuancé par le fait que les nominalisations semblent conserver une trace de l'aspect verbal dynamique, en ce qu'elles décrivent des états épisodiques et impliquent un ancrage événementiel, se démarquant ainsi des noms statifs prototypiques (possession, connaissance, liberté, etc.).

La parenté sémantique repérée, au-delà du décalage aspectuel apparent, entre noms statifs et verbes dynamiques justifie rétrospectivement l'hypothèse déverbale : que le nom, en dépit de sa stativité, garde un lien avec la dynamicité verbale, tandis que le verbe, bien que dynamique, met en saillance un état corrélé permet de justifier l'idée que le nom est dérivé du verbe. L'hypothèse déverbale a pour mérite de contraindre l'analyse du rapport entre verbes et noms, et de mettre en lumière les spécificités des verbes et des noms quant à leur dynamicité et stativité respectives.

Plus généralement, l'analyse des paires $\mathrm{V}$ dynamique / $\mathrm{N}$ statif nous renseigne sur les différents modes de transfert d'aspect dans les nominalisations. Si la préservation absolue des propriétés aspectuelles dans les opérations de dérivation n'est pas toujours vérifiée, il ne semble pas non plus y avoir de rupture complète entre bases et dérivés. Le processus de nominalisation n'est pas nécessairement transparent. Il peut induire certains changements, mais ceux-ci ne sont pas arbitraires : ils obéissent à certaines spécificités sémantiques des unités sources. En ce sens, la nominalisation peut être envisagée comme un révélateur des particularités aspectuelles des bases de dérivation.

\section{Références}

Alexiadou, A. (2001). Functional Structure in Nominals : Nominalization and Ergativity. Amsterdam / Philadelphia : John Benjamins.

Anscombre, J.-C. (1995). Morphologie et représentation événementielle : le cas des noms de sentiment et d'attitude. Langue Française, 105, 40-54.

Anscombre, J.-C. (2003). Psych-Nouns in French : Semantics and Object Classes. Language Research, special issue, $55-76$.

Anscombre, J.-C. (2005). Les deux périphrases nominales un $N$ en train / un $N$ en cours : essai de caractérisation sémantique. In Bat-Zeev Shyldkrot, H. \& Le Querler, N. (éds), Les périphrases verbales, Amsterdam : John Benjamins, 103-117.

Barque L., Huyghe R., Jugnet A. \& Marín R. (2009). Two types of deverbal activity nouns in French. Proceedings of the 5th International Conference on Generative Approaches to the Lexicon, Pisa, 169-175.

Beauseroy, D. (2009). Syntaxe et sémantique des noms abstraits statifs. Des propriétés verbales et adjectivales aux propriétés nominales. Thèse de doctorat, Nancy-Université. 
Binnick, R. (1969). Studies in the Derivation of Predicative Structures. Doctoral dissertation, University of Chicago.

Carlson, G. (1980). Reference to Kinds in English. New York / London : Garland.

Dell, F. (1979). La morphologie dérivationnelle du français et l'organisation de la composante lexicale en grammaire générative. Revue Romane, XIV-2, 189-216.

Dowty, D. (1979). Word Meaning and Montague Grammar. Dordrecht : Reidel Publishing Company.

Flaux, N. \& Van de Velde, D. (2000). Les noms en français : esquisse de classement. Paris : Ophrys.

Fradin, B. (2009). Stative Predicates and Nominalizations in French. Chronos 9, Paris, septembre 2009.

Grimshaw, J. (1990). Argument Structure. Cambridge, MA : The MIT Press.

Gross, M. (1975). Méthodes en syntaxe. Le régime des constructions complétives. Paris : Hermann.

Gross, M. \& Kiefer, F. (1995). La structure événementielle des substantifs. Folia Linguistica Historica, 16, 43-65.

Haas, P. (2009). Comment l'aspect vient aux noms. Les propriétés aspectuelles des noms à l'épreuve des restrictions de sélection imposées par certaines prépositions. Thèse de doctorat, Université de Lille 3.

Haas, P., Huyghe, R. \& Marín, R. (2008). Du verbe au nom : calques et décalages aspectuels. In Durand, J., Habert, B. \& Laks, B. (éds), Congrès Mondial de Linguistique Française-CMLF'08, Paris : Institut de Linguistique Française, 2051-2065.

Haas, P. \& Huyghe, R. (à paraître). Les propriétés aspectuelles des noms d'activités. Cahiers Chronos, 21.

Huyghe, R. \& Marín, R. (2007). L’héritage aspectuel des noms déverbaux en français et en espagnol. Faits de Langues, 30, 265-274.

Kelling, C. (2003). French Psych Verbs and Derived Nouns. In Butt, M. \& King, T.H. (éds), Nominals. Inside and out, Standford : CSLI, 151-179.

Kerleroux, F. (2008). Des noms indistincts. In Fradin, B. (éd.), La raison morphologique. Hommage à la mémoire de Danielle Corbin, Lingvisticae Investigationes Supplementa 27, Amsterdam / Philadelphia : John Benjamins, 113132.

Martin, F. (2008). Les prédicats statifs. Etude sémantique et pragmatique. Bruxelles : De Boeck Duculot.

Meinschaefer, J. (2003). Nominalization of French Psychological Verbs. Syntactic Complements and Semantic Participants. In Quer, J., Schroten, J., Scorretti, M., Sleeman, P. \& Verheugd, E. (éds), Selected Papers from 'Going Romance’, Amsterdam : Benjamins, 235-250.

Pustejovsky, J. (1995). The Generative Lexicon. Cambridge Mass. : The MIT Press.

Ruwet, N. (1972). Théorie syntaxique et syntaxe du français. Paris : Le Seuil.

Ruwet, N. (1995). Les verbes de sentiment peuvent-ils être agentifs ? Langue Française, 105, 28-39.

Van de Velde, D. (1995). Les verbes dits «psychologiques » revus à la lumière des noms correspondants. Revue de Linguistique Romane, 233-234, 67-97.

Verkuyl, H. (1989). Aspectual classes and aspectual composition . Linguistics and Philosophy, 12, 39-94.

\footnotetext{
${ }^{1}$ Cette étude prolonge un ensemble de travaux consacrés à l'héritage aspectuel des noms déverbaux en français (Huyghe \& Marín, 2007, Haas et al., 2008, Barque et al., 2009, Haas \& Huyghe, à paraître). Elle s'inscrit dans le cadre d'un projet de recherche portant sur l'analyse sémantique des nominalisations - projet Jeunes Chercheurs NOMAGE (ANR-07-JCJC-0085-01). Le travail présenté ici a bénéficié des commentaires de P. Haas, R. Marín, L. Barque et D. Amiot, que nous remercions.

${ }^{2}$ Certains noms, comme signification, privilégient l'interprétation non aspectuelle. L'acception stative n'est pas exclue : on la rencontre dans des expressions comme la capacité de signification des images, un événement d'une grande signification. Mais l'interprétation comme « contenu de signification » semble dominante dans l'usage - ou éventuellement indissociable de la lecture stative, auquel cas l'emploi du nom est sous-déterminé.
} 


\begin{abstract}
${ }^{3}$ Dans le cas d'ignorance, on peut considérer que la base est l'adjectif ignorant, auquel cas on n'a pas véritablement affaire à une nominalisation partielle. Reste qu'on peut se demander pourquoi il n'existe pas d'*ignoration, *ignorage ou *ignorement construit sur l'acception dynamique du verbe. Si on estime que la base est verbale, on est face à deux options. Soit on considère que -ance se définit comme un suffixe statif, et alors il faut expliquer pourquoi le verbe sélectionne un tel suffixe - et non un autre, plus neutre, ou éventuellement dynamique, en lien avec l'acception dynamique du verbe. Soit on considère que -ance est, à l'instar de -tion et -ment, transparent aspectuellement, auquel cas il faut justifier qu'ignorance est un nom statif, et non dynamique. Il semble donc nécessaire, dans tous les cas de figure, de se munir d'une hypothèse sémantique sur la base verbale ignorer, pouvant expliquer pourquoi le nom corrélé ne reprend pas l'acception dynamique du verbe.
\end{abstract}

${ }^{4}$ Plus généralement, le problème qui se pose à nous ici est que de nombreux tests employés pour mettre en évidence la dynamicité des verbes portent en fait sur l'agentivité du sujet. Tel est le cas notamment de la tournure injonctive Arrête de $V(y)$ ! et des structures causatives forcer $x \grave{a} V(y)$, faire $V x$ ou faire Vy par $x$.

${ }^{5}$ Un parallèle peut être établi ici avec les verbes de déplacement contourner, longer, traverser, etc., dont le sens est principalement dynamique, mais qui admettent également une lecture non agentive (e.g. La route (contourne / longe/ traverse) la forêt). Il nous semble peu intuitif de traiter ces verbes comme des cas de polysémie [ \pm dynamique] au même titre que admirer ou ignorer. Il est préférable de les considérer comme des verbes intrinsèquement dynamiques, dont la signification spatiale induit un emploi non agentif. Reste qu'à la différence d'amuser et consorts, les verbes de déplacement ont des nominalisations dynamiques, et non statives - ce qui peut en l'occurrence étayer l'hypothèse d'un sens essentiellement dynamique (cf. Fradin, 2009). Cependant, le décalage apparent dans le cas d'amusement, découragement et énervement peut s'expliquer par la structure événementielle de ces verbes, qui associent l'action à un état résultant (cf. § 4). Cette spécificité ne concerne pas les verbes de déplacement, ce qui peut expliquer leur différence de nominalisation.

${ }^{6}$ Significativement, les étymons latins (en -io) des noms français en -tion analysés comme déadjectivaux ont, contrairement à ce qui est le cas en français, une forme verbale correspondante (e.g. adaequatio-adaequare, abjectioabicere, praecisio-praecidere). On peut y voir la marque d'un suffixe fondamentalement lié au domaine verbal.

${ }^{7}$ On rejoint ici une distinction traditionnelle entre verbes agentifs et verbes résultatifs (cf. Dowty, 1979, Martin, 2008). Notons que les verbes « agentifs », plus facilement compatibles avec les structures testant l'agentivité du sujet, n'ont pas systématiquement un sujet agentif - celui-ci peut être non animé non humain, donc dénué du rôle d'agent (e.g. Cette situation l'a (bouleversé / chamboulé / étonné / exaspéré), cf. § 2.3). Il s'agit donc plutôt de distinguer des tendances : les verbes « agentifs » se démarquent des « résultatifs » en ce que leurs sujets tendent à correspondre plus fréquemment à des agents. Nous retenons ici l'idée que certains verbes à expérienceur objet mettent en saillance une action (fréquemment associée à un agent), tandis que d'autres ont tendance à la laisser à l'arrière-plan. 